\title{
EINE PHÖNIZISCHE BRONZESCHALE AUS DEM SUDAN
}

Von Angelika Lohwasser

In den Wintermonaten 1912/13 leitete der Brite F. Ll. Griffith (Oxford) eine Ausgrabung in Sanam, einem Ort nahe dem Gebel Barkal im Sudan. Heute ist das antike Gelände von der modernen Provinzhauptstadt Merawi eingenommen; auch schon zu Griffiths Zeiten begann die Stadt bereits über die Reste der alten Kultur zu wachsen. Sanam liegt in dem Gebiet, in dem der Nil für ca. $300 \mathrm{~km}$ etwa von Nord nach Süd, und nicht wie sonst in seinem 6672 km langem Lauf von Süd nach Nord fließt. Prägend für die Umgebung ist sicherlich der Gebel Barkal, ein freistehender Tafelberg, der als markanter und von weitem sichtbarer Punkt eine Landmarke darstellt. Schon früh wurden an diesem Berg Heiligtümer angelegt; in meroitischer Zeit ist das Gelände zwischen dem Gebel Barkal und der Fruchtlandgrenze mit Tempeln und Palästen übersät. Südlich des Berges liegen zwei Gruppen von Pyramiden, die in die Zeit von ca. 300-270 bzw. 90-50 v. Chr. datiert werden.

In der Nähe des Gebel Barkal muß die Stadt Napata liegen, die als erste Hauptstadt des Reiches von Kusch (ca. 800 v. Chr.-330 n. Chr.) angesehen wird, aber noch nicht lokalisiert ist. Das Reich von Kusch, das sich von Unternubien bis mindestens zum Zusammenfluß von Weißem und Blauem Nil (bei der heutigen Hauptstadt des Sudan, Khartoum) ersteckte, wird in die napatanische und die meroitische Periode geteilt. Der Wendepunkt wird an der Verlegung des königlichen Friedhofes vom napatanischen Raum nach Meroe (um 270 v. Chr.) festgemacht. Die Hauptstadt mit Sitz der Verwaltung wird wohl schon viel früher, spätestens in der Zeit des Aspelta (um 590 v. Chr.) nach Meroe verlegt worden sein.

Im ersten Augenblick verwirrend ist die Tatsache, daß Griffith von seiner Ausgrabung in „Napata“ spricht. Gemeint ist dabei allerdings das Ruinengebiet rund um die alte Hauptstadt, zu der Griffith auch die Königsfriedhöfe von El Kurru und Nuri

\footnotetext{
1 Bald nach Aspelta dürfte der Tempel als eine Uschebtimanufaktur genutzt worden sein. Uschebtis und Modeln dafür wurden gefunden. Aus meroitischer Zeit datieren
}

und natürlich die Bauten am Gebel Barkal zählt. Sanam konnte bisher nicht mit der Stadt Napata identifiziert werden.

Griffith legte zunächst den Tempel für „Amun, Stier von Nubien" frei, einen Sakralbau, der nach der klassisch ägyptischen Raumordnung konzipiert ist. Der Tempel wurde in der Zeit des kuschitischen Königs Taharqo (690-664 v. Chr.) gebaut; ein kleiner Schrein von Aspelta (ca. 593-568 v. Chr.) ist die letzte an diesem Bau nachgewiesene Maßnahme. Bald danach dürfte der Tempel auch nicht mehr in Betrieb gewesen sein, es deuten keine Inschriften oder archäologische Relikte auf eine sakrale Nutzung nach Aspelta hin. ${ }^{1}$ In der Nähe des Tempels befand sich auch das sogenannte „Schatzhaus“, eine Reihe von Kammern mit Säulen, aus denen immer wieder Kleinfunde geborgen wurden. Auch in diesem Fall sowie bei den wenigen Resten der antiken Stadt geht Griffith von einer Blüte in der Zeit zwischen den Königen Piye (747-712 v. Chr.) und Aspelta aus.

Der dritte Altertümerplatz in Sanam, der ebenfalls von Griffith ausgegraben wurde, ist ein Friedhof, der aus ca. 1700 Gräbern besteht. Zeitlich setzt Griffith den Friedhof ebenfalls in die Periode zwischen den Königen Piye und Aramatelqo (ca. 568-555 v. Chr.). Wie Griffith selbst in seinem Grabungsbericht (GRIFFITH 1923) schreibt, ist Sanam der einzige Friedhof in der Nähe der antiken Hauptstadt Napata, der nicht-königliche Gräber beherbergt und der ausgegraben wurde. Dieses Bild hat sich bis heute nur wenig verändert, jedoch wurden in den letzten Jahren einige kleinere Unternehmungen zu diesem Thema unternommen: in Hillat el-Arab, einem Ort zwischen dem Gebel Barkal und dem ältesten Königsfriedhof, El Kurru, sowie in der Nähe der Pyramidenfelder am Gebel Barkal selbst wurden einige Privatgräber ausgegraben. ${ }^{2}$

Insgesamt wurden von Griffith ca. 1550 Gräber untersucht, einen kleinen Teil konnte er nicht aus- eine Inschrift, Keramikscherben sowie die Wiedererrichtung einer Ziegelmauer (GRIFFITH 1922: 75).

2 Vincentelli-Liverani 1997; Berenguer 1999. 
graben, da er im Hof des Krankenhauses bzw. unter der Straße zum Sitz des Provinzgouverneurs liegt. Die meisten Gräber wurden bereits in der Antike geplündert, oft auch die Skelette zerstört. Trotz allem gelang es Griffith, eine Reihe von Funden aus den Gräbern zu bergen.

Das Ägyptische Museum Berlin unter dem damaligen Direktor Heinrich Schäfer war einer der vielen Sponsoren der Oxford Expedition to Nubia. ${ }^{3}$ Aus diesem Grund wurde Berlin auch bei der Fundteilung bedacht und so kam eine beträchtliche Menge von Kleinfunden in das Ägyptische Museum. ${ }^{4}$ Der Großteil der Objekte sind Perlen, Amulette, Skarabäen und Schmuckteile aus Bronze, Muscheln und anderen Materialien, jedoch befinden sich außer Gefäßen aus Ton auch einige aus Metall und Stein darunter. Nur wenige Stücke fallen aus diesem Rahmen: vier Spiegel aus Bronze, eine Opfertafel aus Ton, eine Kopfstütze aus Elfenbein, ein Salblöffel aus Fayence, ein Reibstein aus Quarz und ein Schminkgriffel aus Glas.

Das Augenmerk soll nun auf eine Bronzeschale gelenkt werden, die in einem Grab des Friedhofes von Sanam gefunden wurde und sich heute im Ägyptischen Museum Berlin befindet. Die Schale trägt die Inventarnummer 2989 und stammt aus dem Grab Nr. 850. GrifFiTh (1923: 160) bemerkt zu dem Grab lapidar: „850. Rect. bricked. Bronze bowl XVI (III b). Berlin.“ Es handelt sich also um ein rechteckiges Grab, das mit Ziegeln verkleidet wurde; eine auf diesem Friedhof sehr häufige Grabform. Leider gibt sein Bericht keine Auskunft, um welche Art der Bestattung es sich handelt. Nach der Zusammenstellung von Griffith sind in solchen Gräbern aber vor allem Mumien bzw. Strecker zu finden, allerdings sind auch einige Hocker nachgewiesen (GRIFFITH 1923: 78). Auch das Geschlecht und das Alter des Verstorbenen werden bei Griffith nicht genannt. Nach dem Bericht von Griffith ist in dem Grab nur eine Bronzeschale gefunden worden. Das kann allerdings nicht mit Sicherheit angenommen werden, da Griffith die Funde, die nicht direkt in der Grabkammer gefunden wurden, fast nie angibt. Wir müssen nun als Arbeitshypothese annehmen, daß direkt beim Skelett nur die Bronzeschale 2989 gelegen ist.

Bei der Zusammenstellung der Funde schreibt Griffith über unsere Schale unter der Rubrik „III. Shallow bowls and cups" folgendes (GRIFFITH 1923: 92): „Heavy, rim hammered down, four groups of three very shallow bosses in a horizontal row hammered in from the outside near the foot, and four groups of four each below the rim, 850." Er verzeichnet also keine Dekoration, jedoch einige von außen eingeschlagene Beulen. Bereits bei der ersten Restaurierung nach dem Eingang des Objektes wurde festgestellt, daß diese Beulen die Körper von Rindern waren, die durch Linien in die Schale eingezeichnet waren. Nach Beschädigungen durch den Krieg, die zum Glück nahezu ohne Substanzverlust vonstatten gingen, wurde die Schale unlängst erneut von Frau R. Lehmann restauriert. ${ }^{5}$

INV. Nr. 2989 (= Z-545) (Abb. 1-5)

Durchmesser 180 mm; Dicke 2,4-1,7 mm

Flache Schale, Teil einer Kugel, ohne besonders abgesetzten Rand. Außen ohne Dekoration. Innendekoration mit eingravierten Linien.

Mitte: Kreis (Ø $25 \mathrm{~mm}$ ) mit kleinem Punkt in der Mitte, in den Kreis ragen vier Halbkreise, die mit einer Schraffur verziert sind. An diesen Kreis anschließend ein zweiter Kreis, auf den außen 26 schildförmige Blütenblätter gesetzt sind.

Unterer Fries: Um die Mittelverzierung umlaufend ein Fries, der oben und unten mit einer durch schräge Punkte bezeichneten Linie begrenzt ist. Darstellung von vier Rindern, die nach links schreiten, ihren Kopf aber zurück (nach rechts) gedreht haben. Von den vier Rindern sind zwei gut erhalten, eines zum Teil korrodiert, eines durch Korrosion
${ }^{3}$ Aus den Akten des Zentralarchives der Staatlichen Museen zu Berlin war zu erfahren, daß die Sachverständigenkommission der Ägyptischen Abteilung am 3. 1. 1913 den Beschluß faßte, die Ausgrabung Griffiths mit $2500 \mathrm{M}$ zu unterstützen. Weiters geht aus den Akten hervor, daß diese Summe am 28. 4. 1913 als L 122.2.7.in London ausgezahlt wurde. Leider sind keine Unterlagen über die Fundteilung, den Transport der Objekte und deren Übereignung mehr erhalten.

4 Im Zuge meines Volontariats am Ägyptischen Museum war es mir möglich, die Objekte aus Sanam zu studieren und in eine Datenbank aufzunehmen. Für die immer gezeigte Hilfsbereitschaft danke ich Prof. Dr. K.-H. Priese (Direktor am Ägyptische Museum) und Herrn F. Marohn (Magazinverwalter des Ägyptischen Museums) ganz herzlich. Ohne ihren Beistand wäre mir diese Untersuchung nicht möglich gewesen.

${ }^{5}$ Dankenswerter Weise machte mich Herr Prof. Dr. Priese auf diese Schale aufmerksam und empfahl mir, sie im nun restaurierten und somit handhabbaren Zustand genauer zu untersuchen. Herr Prof. Dr. D. Wildung danke ich für die Publikationserlaubnis. 

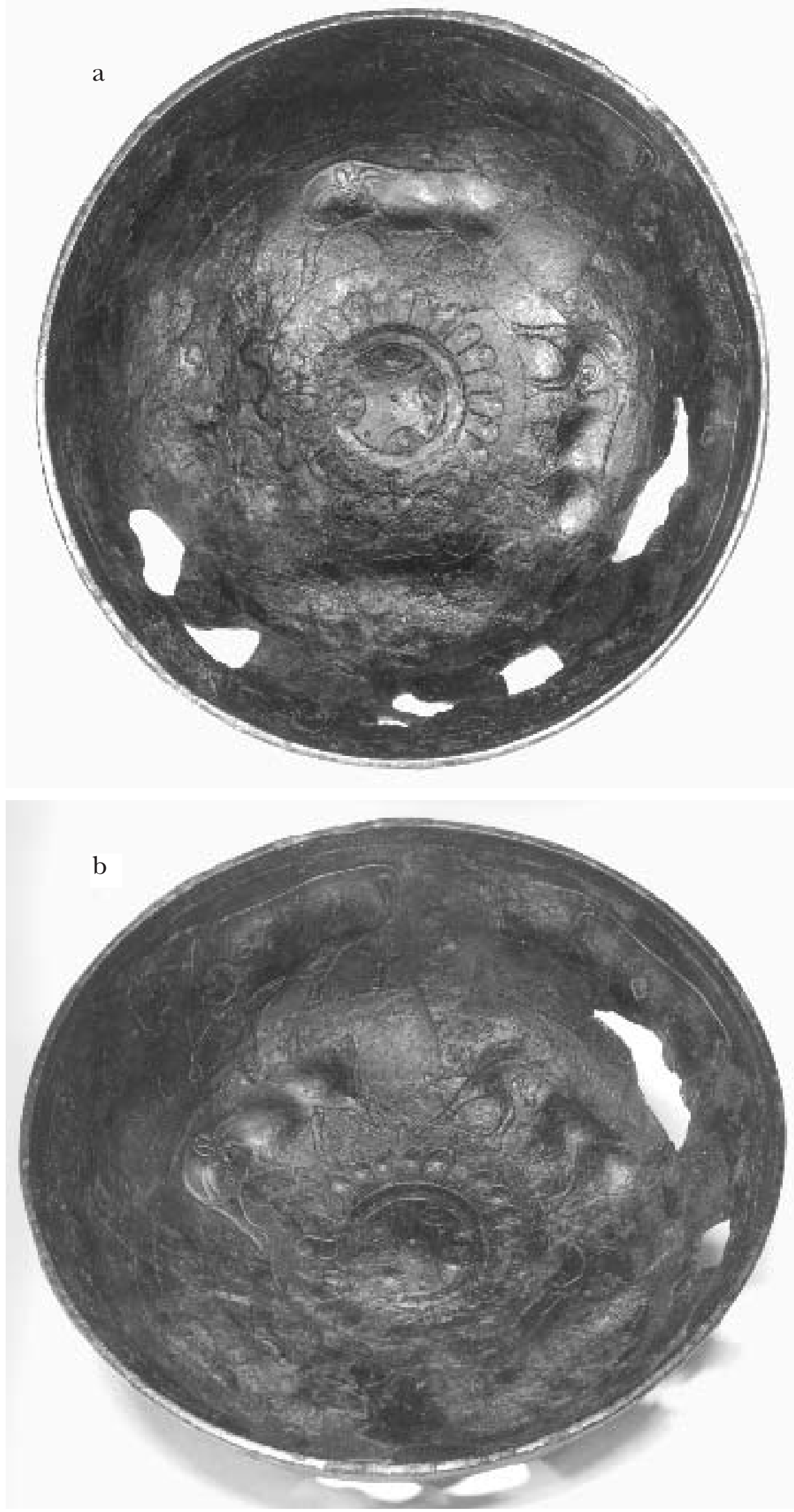

Abb. 1a, b Bronzeschale, Ägyptisches Museum Berlin, Inv. Nr. 2989. Foto: M. Büsing 

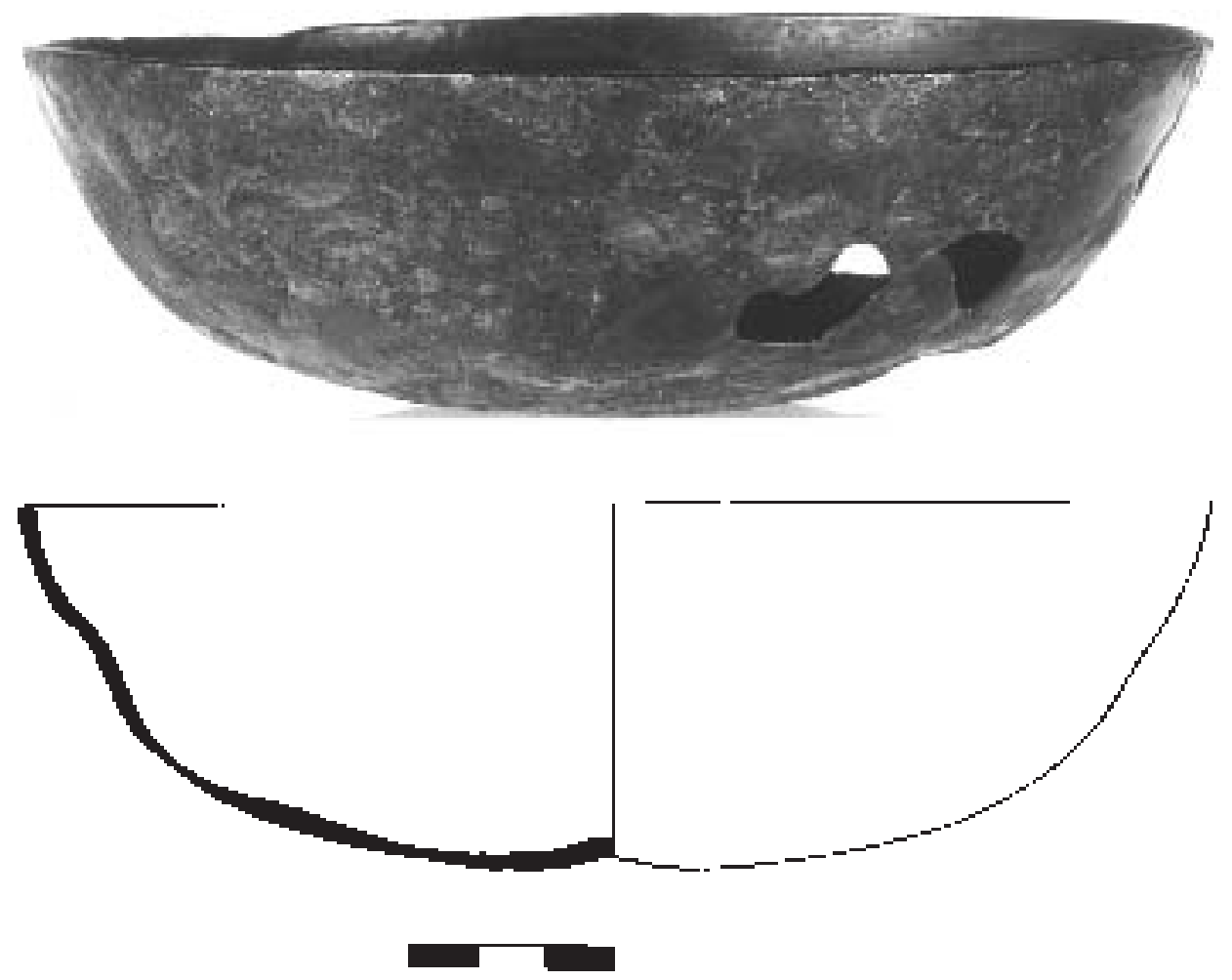

Abb. 2 Bronzeschale, Ägyptisches Museum Berlin, Inv. Nr. 2989. Foto: M. Büsing, Zeichnung: I. Gerullat

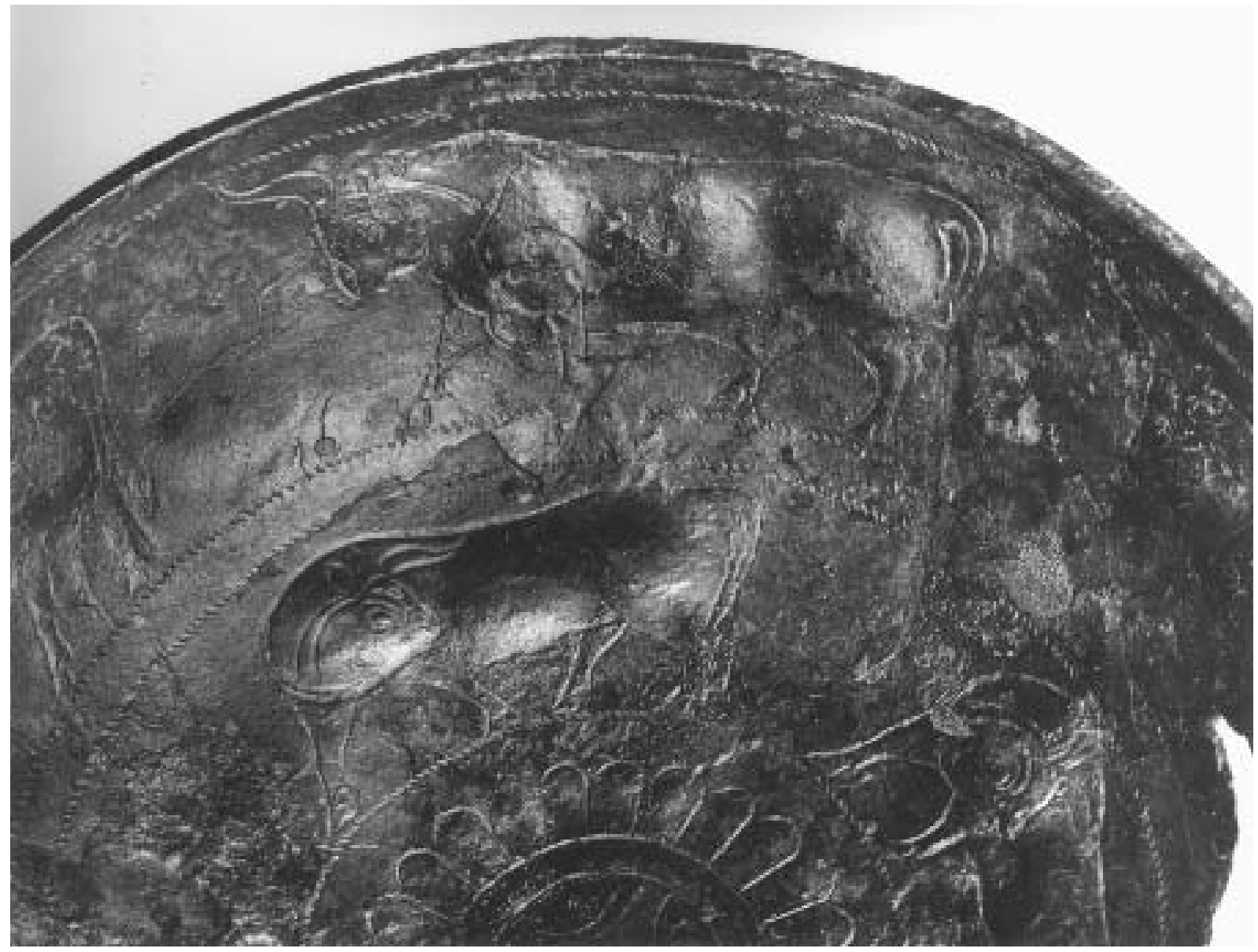

Abb. 3 Bronzeschale, Ägyptisches Museum Berlin, Inv. Nr. 2989. Foto: M. Büsing 


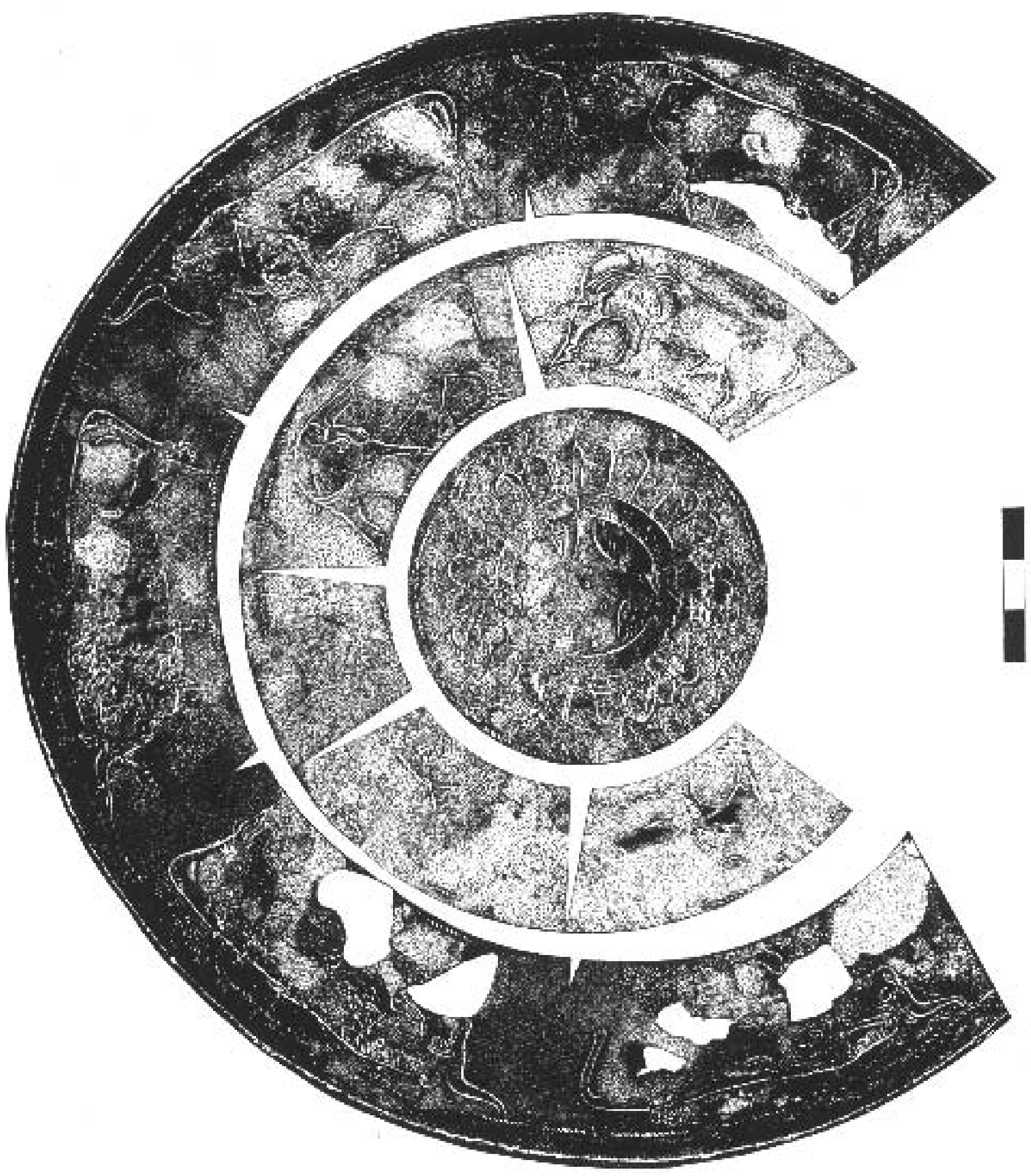

Abb. 4 Bronzeschale, Ägyptisches Museum Berlin, Inv. Nr. 2989. Zeichnung: I. Gerullat 


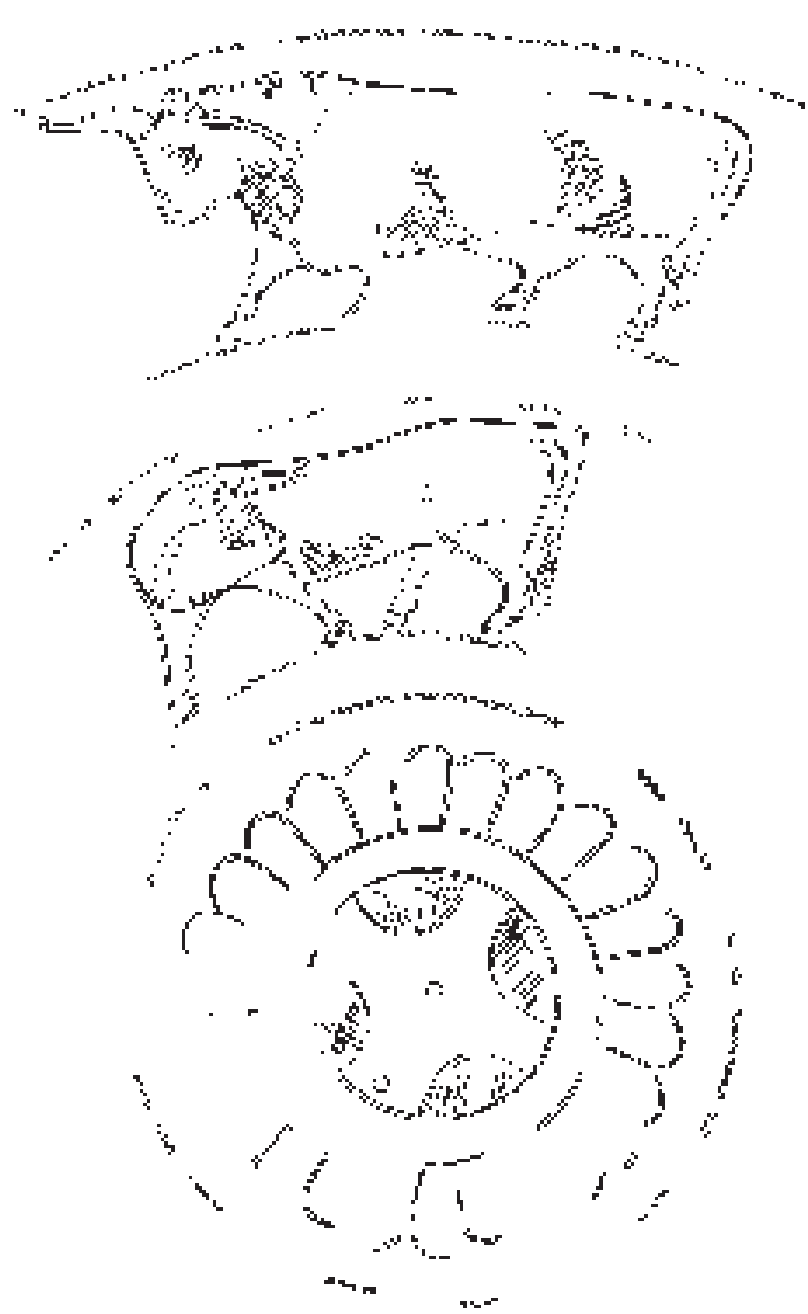

Abb. 5 Bronzeschale, Ägyptisches Museum Berlin, Inv. Nr. 2989. Zeichnung: I. Gerullat

fast nicht mehr sichtbar. Die Beschreibung basiert auf den beiden gut erhaltenen Darstellungen. Das Rind wirkt in die Länge gezogen, Kopf, Bauch und Hinterteil geben durch kleine Wölbungen (von außen eingeschlagene Beulen) einen plastischen Eindruck wieder. Körper, Schwanz und Beine sind durch einfache Linien gekennzeichnet, nur am Kopf sind Details eingezeichnet: das Auge mit einer Braue, zwei Halsfalten, zwei Ohren, allerdings nur ein Horn. Die Schnauze erscheint etwas verkürzt. Die Beine sind steif, beide Vorderbeine gespreizt, die Hinterbeine in Schrittstellung. Bei den Hufen sind die Afterklauen naturalistisch abgesetzt. Der Schwanz macht einen Bogen, indem er vom Körper zunächst etwas absteht, sich dann aber an die Beine schmiegt. Die Schwanzspitze hat eine Innenzeichnung durch Längslinien.

Oberer Fries: Über dem eben beschriebenen Fries befindet sich noch ein zweiter, ebenfalls oben und unten mit einer durch schräge Punkte bezeichneten Linie begrenzt. Dargestellt sind fünf Rinder, die nach links schreiten und auch nach links blikken. Auch hier sind zwei Rinder gut erhalten, von zweien ist durch eine größere Fehlstelle nur der obere Teil zu sehen, ein Rind ist stark korrodiert. Die Art der Darstellung entspricht den Rindern des unteren Frieses, ebenso die eingeschlagenen Beulen. Die Kopfpartie ist jedoch detaillierter wiedergegeben: an der Brust sind drei Fettpölster sowie ein Fell durch feine Querstriche eingezeichnet. Auf dem Kopf und am Nacken sind ebenfalls Haare durch sehr feine Linien angedeutet. Die sichtbare Schulter sowie der Hals bilden Hautfalten (einfache Linien). Am Kopf sind das Auge, die Braue und Nüstern gezeigt, das Maul selbst ist nur durch den Verlauf der Umrißlinie, nicht jedoch durch eine Innenzeichnung wiedergegeben. Bei fast allen Rindern sind beide Ohren zu sehen, nirgends jedoch mehr als ein Horn. Dieses Horn ist mit zwei schwungvollen Linien nach vorne gebogen. Über dem oberen Abschluß des Frieses ist ein $6 \mathrm{~mm}$ breiter Rand, der nicht verziert ist.

Schalen mit Rinderdarstellungen sind im kuschitischen Kulturraum nur selten belegt. Etwa zeitgleich mit dem Grab in Sanam ist das Grab in El Kurru Ku. 55 zu datieren, in dem zwei Fayenceschalen mit Rindern in leicht erhabenem Relief gefunden wurden. ${ }^{6}$ Auch hier schreiten Rinder in einem Fries um eine runde Mittelgestaltung. Bei der Schale MFA 24.1089 ist in dem Kreis eine unter Papyruspflanzen liegende Kuh gezeigt, den Mittelpunkt der anderen Schale bildet eine Rosette. Auf dem Gefäß aus Boston sind die Stiere nach links schreitend gezeigt. Ein nach vorn gestrecktes Horn sowie ein Ohr sind sichtbar. Die Mähne am Nacken ist mit vier bzw. fünf Strähnen wiedergegeben. Am Kopf sind Augen und Brauen sowie Maul und Nüstern mit wenigen Linien eingezeichnet. Der Kopf ist gerade nach vorn gestreckt, der Hals wirft keine Falten wie auf der Bronzeschale aus Sanam. Der Schwanz hängt gerade herunter und ist nicht wie auf unserer Schale vom Körper wegstehend. Auch Penis und

${ }^{6}$ Dunham 1950: 93, fig. 31e, pl. LXIV A., B. Datiert wird das Grab einer Frau in die Zeit des Königs Piye. 
Hoden der Stiere sind dargestellt, ebenso die Hufe mit den Afterklauen. Die Rinder schreiten auf einem kordelartig gedrehten Band. In der Mitte ist ein Medaillon mit einer unter vier Papyruspflanzen liegenden Kuh. Ihr Kopf wirkt leicht gedreht, sodaß beide Hörner sichtbar sind. Eine unter Papyruspflanzen liegende Kuh ist manchmal in der 3. Zwischenzeit in Ägypten dargestellt. ${ }^{7}$

Die zweite Schale, deren heutiger Standort nicht zu ermitteln war, trägt ebenfalls einen Fries mit wahrscheinlich vier Rindern, ${ }^{8}$ die auf einem kordelartigen Band schreiten. Die Art der Darstellung dürfte der anderen Fayenceschale entsprechen. In der Mitte des Bodens ist eine Scheibe, um die herum sich zwölf Blütenblätter zu einer Rosette gruppieren.

Ungewöhnlich ist die Art der Darstellung von Stieren mit nur einem Horn. Die aus Nubien bekannten Abbilder von Rindern lassen sich nicht damit vergleichen, ${ }^{9}$ auch die originär ägyptischen Belege zeigen die Tiere in einer anderen Darstellungsart. ${ }^{10}$ Dieses hervorstechende ikonographische Detail ist jedoch bei Rinderdarstellungen im Flachbild aus dem vorderasiatischen Raum belegt. ${ }^{11}$ Aus dem Palast des Assurnasirpal (883-869 v. Chr.) in Nimrud konnten einige Reliefplatten 1855 für das Vorderasiatische Museum Berlin angekauft werden. Dabei befindet sich auch der linke Teil der Szene einer Stierjagd. ${ }^{12}$ Leider ist nur die vordere Hälfte eines laufenden und das vordere Viertel eines bereits gefallenen Tieres erhalten. Doch die ikonographischen Übereinstimmungen sind bemerkenswert: bei beiden Tieren ist nur ein nach vorne gerichtetes Horn sichtbar, das so wie auf der Bronzeschale aus zwei geschwungenen Linien gebildet ist. Der Kopf ist detailreicher gestaltet, jedoch ist dabei die Größe des
Reliefs zu beachten. ${ }^{13}$ Auch auf der Schale selbst sind die größeren Tiere des oberen Registers mit einer detaillierteren Innenzeichnung versehen als die kleineren des unteren Registers. Auf dem Relief des Assurnasirpal sind das Auge mit stark modellierten Brauen, Nüstern, Maul und Zunge sowie beide Ohren deutlich wiedergegeben. Auf der Brust und am Nacken sind Haare des Felles mit feinen Linien angedeutet. Die Vorderbeine sind im Sprung beide nach vorn gestreckt, jedoch sind auch hier die Hufe (deutlich als Paar zu erkennen) sehr naturalistisch gezeichnet und die Afterklauen wiedergegeben, auch die Muskeln der Beine sind dargestellt. Im Palast des Assurnasirpal sind noch weitere ähnliche Rinderdarstellungen gefunden worden. ${ }^{14}$ Rinder in der eben beschriebenen Ikonographie sind auch auf Elfenbeinfragmenten, die ebenfalls aus Nimrud stammen, belegt. Dabei sind vor allem Rinder mit nach hinten gewendetem Kopf $\mathrm{zu}$ finden. ${ }^{15}$ Diese Kühe lecken ein Kalb, das sie säugen. Obwohl diese Szene nicht auf unserer Bronzeschale dargestellt ist, so ist doch die Ikonographie und die Haltung der Rinder zu vergleichen. Auch eine Bronzeschale mit Darstellungen von Rindern, Löwen, Gazellen und Fabeltieren wurde in Nimrud gefunden. ${ }^{16}$ Die Dekoration der Schale ist anders aufgebaut als bei unserer, doch entspricht auch hier die Ikonographie der der Schale aus Sanam. Es ist wohl anzunehmen, daß das Motiv der schreitenden Rinder in dieser besonderen Form, wie es auf unserer Bronzeschale und den beiden Fayenceschalen aus $\mathrm{Ku} .55 \mathrm{zu}$ finden ist, aus Vorderasien stammt. ${ }^{17}$

Diese Art der Gestaltung der Rinder übernahmen die Phönizier, um orientalisierendes Kunsthandwerk herzustellen. Besonders die Bronzearbei-

7 Friedmann 1998: 234, Nr. 138, mit den Belegen in der Anm. 209.

8 Ein Teil der Schale fehlt und das Foto in Dunham 1950: pl. LXIV B. ist so hell, daß man nur ein Tier erkennen kann.

9 Hofmann \& Tomandl 1987: 34-38, 135-144.

10 Z. B. Brewer, RedFord \& RedFord 1994: 82-89; OSBORNE 1998: 194-196.

11 Bei der Suche nach Literatur waren mir besonders Frau Dr. Nadja Cholidis und Herr Dr. Lutz Martin (Vorderasiatisches Museum) behilflich, denen ich ganz herzlich danken möchte. Es soll jedoch darauf hingewiesen werden, daß die Vfn. Ägyptologin ist und hier keine für einen vorderasiatischen Archäologen erschöpfende Diskussion der Schale liefern kann. Dieses außergewöhnliche Stück soll im Wesentlichen hier nur vorgestellt werden, eine genauere Einordnung

bezüglich Datierung, Herkunft und Stil mögen die Spezialisten vornehmen.

12 VA 962, Foto in MeYer 1965: Abb. 111, Zeichnung in READE 1985: pl. XLIb, Publikation mit älterer Literatur in Paley \& SobolewsKi 1987: 75-76, pl. 5,25.

13 Gesamthöhe 0,79 m, Größe des laufenden Stieres ca. $0,60 \mathrm{~m}$.

14 Siehe Budge 1914: pl. XII.1., XIX.1., XXIII.2; Paley \& SOBOLEWSKI 1987: 76-77, pl. 5,26.

15 Z. B. Hermann 1992: pl. 28, insbesonders Nr. 132-134. Sie stammen aus der Zeit des Nachfolgers von Assurnasirpal II, Salmanassar III.

16 LAYARD 1853: pl. 60.

17 So schon Hofmann \& Tomandl 1987: 34. Hölbl 1979: 307 möchte die Frage, in welche Richtung der Einfluß verlief - ob von Kusch nach Phönizien oder umgekehrt - offen lassen. 
ten aus Phönizien waren ein beliebtes Handels- und Tributgut. ${ }^{18}$ Unter diesen Bronzewerken, die fast ausschließlich außerhalb Phöniziens gefunden wurden, befanden sich auch dekorierte Schalen. Eine Gruppe dieser Schalen wurde „bull-bowls“ genannt, es handelt sich ausschießlich um Gefäße mit Rinderfriesen. Unter diesen Schalen fand sich eine sehr nahe Parallele zu unserer Inv.-Nr. 2989. Sie stammt aus Ägypten. ${ }^{19}$ Eine genaue Herkunft konnte nicht ermittelt werden, die „suggested (but unlikely) provenance is Deir el Bahari“. ${ }^{20}$ Die fünf Rinder am äußeren Fries schreiten nach rechts, die vier am inneren Fries mit zurückgewendetem Kopf nach links. Sie säugen keine Kälber. In der Rosette scheint ebenfalls eine Innenzeichnung aus vier kleinen Halbkreisen zu sein. Die Art der Darstellung ist hier sehr ähnlich der unserer Bronzeschale aus Sanam. Die Blüten der Rosette sowie die Gestaltung der Standlinien mit vielen kleinen Schrägstrichen ist nahezu identisch mit unserer Bronzeschale. Unterschiede sind für mich lediglich beim Schwanz (die Schwanzspitze ist gedreht gezeigt) und in der gegenläufigen Schreitrichtung der Rinder zu erkennen.

Eine weitere vergleichbare Schale befindet sich in der Ortitz-Collection und wurde im Antikenhandel in der Türkei gekauft. ${ }^{21}$ Auch hier ist der äußere Fries mit fünf nach links schreitenden Stieren gestaltet, der innere mit vier Kühen, die ihren Kopf nach hinten wenden, um ihr säugendes Kalb zu lecken. Den Mittelpunkt bildet eine Rosette, deren Innerstes wie im Fall unserer Schale mit einem Kreis und darin hineinragend vier schraffierten Halbkreisen gebildet ist. Die Stiere und auch die Kühe sind stilistisch und ikonographisch mit den Rindern unserer Schale zu vergleichen. Auch hier sind durch von außen eingeschlagene Beulen die Körper der Tiere plastisch gestaltet. Ebenso zeigt die Darstellung mit dem einen, nach vorne wegstehenden geschwungenen Horn, die Fellzeichnung an Hals und Nacken sowie der zunächst wegstehende und dann an den Beinen fast anliegende Schwanz die fast identische Ausführung. Auch die zurückgewandten Kühe sind parallel zu unserer Schale gestaltet. Jedoch wird bei der Schale aus Sanam kein Kalb gesäugt.

Eine weitere ähnliche Schale befindet sich heute im British Museum (BM 134711), die allerdings nicht vollständig erhalten ist. ${ }^{22}$ Die Stiere scheinen nicht so sorgfältig gearbeitet zu sein. Bei den im inneren Fries zurückblickenden Rindern ist heute nicht mehr festzustellen, ob sie ein Kalb säugen oder nicht. Die beiden Schalen (Ortitz-Collection, British Museum) werden von FALsone (1985: 141) in das 9./8. Jh. v. Chr. datiert.

Ebenfalls in diese Gruppe gehört die Schale aus dem Museum Teheran 15192. ${ }^{23}$ Am Fries außen schreiten die fünf Stiere nach links, allerdings ist in diesem Fall der innere Fries undekoriert geblieben. Den Mittelpunkt der Bronzeschale bildet wieder eine Rosette mit dem Kreis, in den vier schraffierte Halbkreise ragen. Weitere Parallelen sind zwei Schalen aus Olympia, die mit schreitenden Rindern in einem ähnlichen Stil wie die Schale aus El Kurru dekoriert sind, ${ }^{24}$ sowie eine aus Mykonos ${ }^{25}$ und eine aus Nimrud. ${ }^{26}$ Auch in Phrygien ist eine dieser Schalen in einem Grab gefunden worden. ${ }^{27}$

In seiner Untersuchung zu den cypro-phönizischen Bronzeschalen datiert MARKOE (1985:153) sämtliche „bull-bowls“ in die 2. Hälfte des 8. Jh. v. Chr. Er begründet die Datierung mit dem Hortfund in Nimrud, der in der Regierungszeit des Sargon II. (722-705 v. Chr.) angehäuft wurde und daher vorher entstanden sein mußte. Sowohl Falsone als auch Culcian datieren die von ihnen besprochenen Schalen etwas früher in das 9./8. Jh. v. Chr.

Durch die reichlich vorhandenen Parallelen hat sich gezeigt, daß die Bronzeschale aus Sanam in die Gruppe der phönizischen „bull-bowls“ gehört. Die Wiederverwendung von fremden Motiven ist ganz typisch für die phönizische Kunst. Besonders ägyptische Elemente wurden gern verwendet, gab es doch

18 FAlsone 1988. Siehe auch Hölbl 1979: 293-322. Eine ältere, aber auf jeden Fall beachtenswerte Untersuchung zu den phönizischen Metallschalen ist Bissing 1923/24.

19 Ehemals Coll. Mac Gregor Nr. 1203, heute im St. Louis Art Museum Nr. 115.1924 (FAlsone 1985: 141, pl. XXIV b; Markoe 1985: 215-216, U2; 344).

${ }^{20}$ MARKoE 1985: 215-216. WALLIS (1898: 70-71) schreibt: „it is said, on good authority, to have formed a part of a Find of objects which belonged to Queen Hatshepsu."

${ }^{21}$ FALsOne 1985: 131-136.

${ }^{22}$ FALSONE 1985: 136-138; Herkunft: möglicherweise Iran.

23 Culcian 1970: 71-73, pl. VIII, 2; Markoe 1985: 216, U3.

${ }^{24}$ Archäolog. Musum Olympia: Inv. Nr. 8555 (MARKOE 1985: 206, G5; FALSONE 1985: 141), Inv. Nr. B6094 (CULCIAN 1970: 69-71, fig. 2; MARKoe 1985: 206, G6).

25 Archäolog. Museum Mykonos, Inv. Nr. 480 (MARKoE 1985: 208, G10).

26 Falsone 1988: 236.

27 Ankara G.T. 57, AtAsoy / Buluc 1982: 157, pl. XXIXa. 
nach Ägypten die längsten und intensivsten Handelskontakte. Aber auch assyrische Motive wurden übernommen und in die Kunstwerke des orientalisierenden Stils der Phönizier aufgenommen. Kunsthandwerk der Phönizier bediente gerne den Geschmack ihrer Handelspartner. Die assyrischen Elfenbeinschnitzereien, die in Nimrud gefunden wurden, stammen oft aus phönizischen Werkstätten. Darum ist es auffällig, daß gerade drei mit Rindern dekorierte Gefäße ihren Weg nach Nubien fanden. Das mag Zufall sein, es kann aber auch mit der besonderen Bedeutung von Rindern für die nubischen Kulturen zusammenhängen. ${ }^{28}$ Vor allem in der meroitischen Periode des Reiches von Kusch scheint das Rind außer dem wichtigsten Opfertier auch noch eine weitergehende sakrale Bedeutung gehabt zu haben. ${ }^{29}$ Gerade für die napatanische Periode fehlen uns aber die häufigen Rinderdarstellungen. Ob daher auf eine bewußte Vorliebe für Rinderschalen in Napata geschlossen werden kann, mag dahingestellt bleiben.

Es bleibt noch zu überlegen, wie die phönizische Bronzeschale in die Hände der Kuschiten gelangt sein könnte. Dabei können verschiedene Wege eingeschlagen werden. Vielleicht befand sich die Schale bereits vor der Eroberung Piyes (728 v. Chr.) in Ägypten - sie könnte als Handelsgut aus Phönizien dorthin gelangt sein - und Piye ließ sie als Kriegsbeute in sein Schatzhaus bringen. In seiner Siegesstele aus dem 21. Regierungsjahr heißt es: „(Z. 153) Und die Schiffe waren beladen mit Silber, Gold, Kupfer, (Z. 154) Leinen, allen Dingen aus Unterägypten, allen Erzeugnissen aus Syrien, allen Essenzen aus dem Gottesland. “30 Mit dieser Beute, zu der vielleicht auch unsere Schale gehört hatte, fuhr Piye mit seiner
Armee südwärts in die Heimat nach Napata. Bereits Kendall (1986: 18) wies darauf hin, daß die Kuschiten unter Piye wahrscheinlich phönizisches Kunsthandwerk als Beute in ihre Heimat genommen haben. Vor allem für die Darstellung der Unterwerfung der unterägyptischen Fürsten im Amuntempel am Gebel Barkal ${ }^{31}$ sieht er die Vorbilder in phönizischen Schalen mit Pferde- und Kampfdarstellungen. Die Kombination der einzelnen Motive sowie der Stil der Pferde seien weniger den ägyptischen Reliefs des Neuen Reiches, als vielmehr dekorierten phönizischen Gefäßen angelehnt. ${ }^{32}$

Andererseits könnte das Gefäß auch mit den Assyrern selbst nach Ägypten gebracht worden sein. Nach einer Phase der Ausdehnung des Neuassyrischen Reiches bis an die Grenzen Ägyptens kam es unter dem König Asarhaddon in den Jahren 674, 671 und 669 zu kriegerischen Auseinandersetzungen zwischen den Assyrern und den Kuschiten in Ägypten..$^{33}$ Unter seinem Nachfolger Assurbanipal kam es zu den entscheidenden Kriegszügen (667/6 bzw. 663 v. Chr.), die mit der Zerstörung Thebens durch die Assyrer und der Vertreibung der Kuschiten endeten. Die Schale kann im wechselvollen Kriegsschicksal ihren Besitzer gewechselt haben.

Nicht zuletzt besteht aber auch die Möglichkeit von direkten Handelskontakten zwischen den Kuschiten und Phöniziern. Es ist zu bedenken, daß sich die kuschitischen Könige der Gunst ihrer Untertanen auch durch Geschenke von Luxusgütern, zu denen diese Schale sicher zählt, versicherten. ${ }^{34}$ Dafür spricht, daß noch weitere Objekte vorderasiatischen Ursprungs in Sanam gefunden wurden. ${ }^{35}$ Unter den Funden, die nach Berlin

${ }^{28} \mathrm{Zu}$ den Rinderdarstellungen in der C-Gruppe HoFMANN \& TOMANDL 1987: 28-29. Besonders hervorzuheben sind die beiden „cattle-bowls“ aus Aniba, die Rinderdarstellungen in außergewöhnlicher Qualität tragen. Rinder in der Kerma-Kultur: Hofmann \& TOMAndL 1987: 32. In der napatanischen Periode des Reiches von Kusch finden sich kaum Rinderdarstellungen, in der meroitischen Periode hingegen sind sie sehr häufig abgebildet (Belege: Hofmann \& TOMANDL 1987: 135-144).

29 Hofmann \& Tomandl 1987: 156-159; Wenig 1993: 218; ONASCH, CH., 1993: 260. Ein bemerkenswerter Beleg dazu ist die „Tabu-Szene“ im Löwentempel von Musawwarat es-Sufra (innere Nordwand, unterer Abschlußstreifen; siehe HINTZE 1971: Tf. 67; WENIG 1993: 111), in der eine Kuh mit einem Tuch fast vollständig verdeckt ist. Möglicherweise soll das Trinken am Euter verborgen werden.

30 Stele Kairo JE 48862, GRIMAL 1981: 180-181

31 B 501, Nordwand (Kendall 1986: fig. 8), B 502, Rückwand des 2. Pylon (Kendall 1986: fig. 9, 10).

32 Kendall 1986: 23, Anm. 34.

33 ONASCH, H.-U, 1994: 16. Siehe zu den assyrischen Eroberungen Ägyptens insgesamt ONASCH, H.-U, 1994.

34 Diese Vermutung äußerte bereits HeIdorn 1994: 127-128. Für das spätere Reich von Meroe als Grundlage der Macht der Könige angenommen und untersucht von Edwards 1996, siehe bes. 40-47. Siehe auch WELSBY 1996: 174-175.

35 Griffith (1923: 89, 124, 169, pl. XXXI.8) erwähnt eine "graeco-phoenician vase of Cyprus" (Oxford, Ashmolean Museum 1921.989) aus dem Grab 1416, zwei „little bottles of very fine fabric, much decayed (1923: 89)“ aus dem Grab 714 sowie einen syrischen Zylinder (HOGARTH 1922: 215-216, pl. XXV; GRIFFITH 1923: 89) aus dem Grab 396. Möglicherweise existieren aber noch weitere phönizische Objekte aus Sanam, die von Griffith aber nicht publiziert wurden. 
gelangt sind, befinden sich drei phönizische Kannen. ${ }^{36}$ Inv.-Nr. 7852 (Abb. 8) und 7871 (Abb. 9) haben den typischen langen, leicht bauchigen Hals, einen kleinen Henkel und einen breiten, nach außen gestülpten Rand („mushroom lipped“). Unter einer abgeschrägten Schulter wölbt sich ein fast eiförmiger Gefäßkörper auf einem kleinen Standring. Beide Kännchen sind rotpoliert. Die Inv.-Nr. 7852 ist auf der Oberseite der Gefäßlippe mit gebogenen Schrägstrichen dekoriert. Diese „mushroom lipped“ Kännchen sind vom 9.-5. Jh. v.
Chr. belegt. ${ }^{37}$ Nach der Typologie der Pilzkännchen von A. Peserico (1996) stammt keines der Kännchen aus dem punischen Raum, da dort viel längere Hälse vorherrschen. Inv. Nr. 7852 kommt aus dem vorderasiatischen Raum und ist 750-680 v. Chr. zu datieren, Inv. Nr. 7871 kann nicht so genau eingeordnet werden. ${ }^{38}$ Als zypro-phönizisch anzusehen ist das Kännchen Inv.-Nr. 7851 (Abb. 6, 7). Es hat ebenfalls einen Henkel, der Gefäßkörper ist fast kugelig mit einer Standfläche. Es ist aus feinem rötlich-ockerfarbenem Ton und trägt am Bauch eine
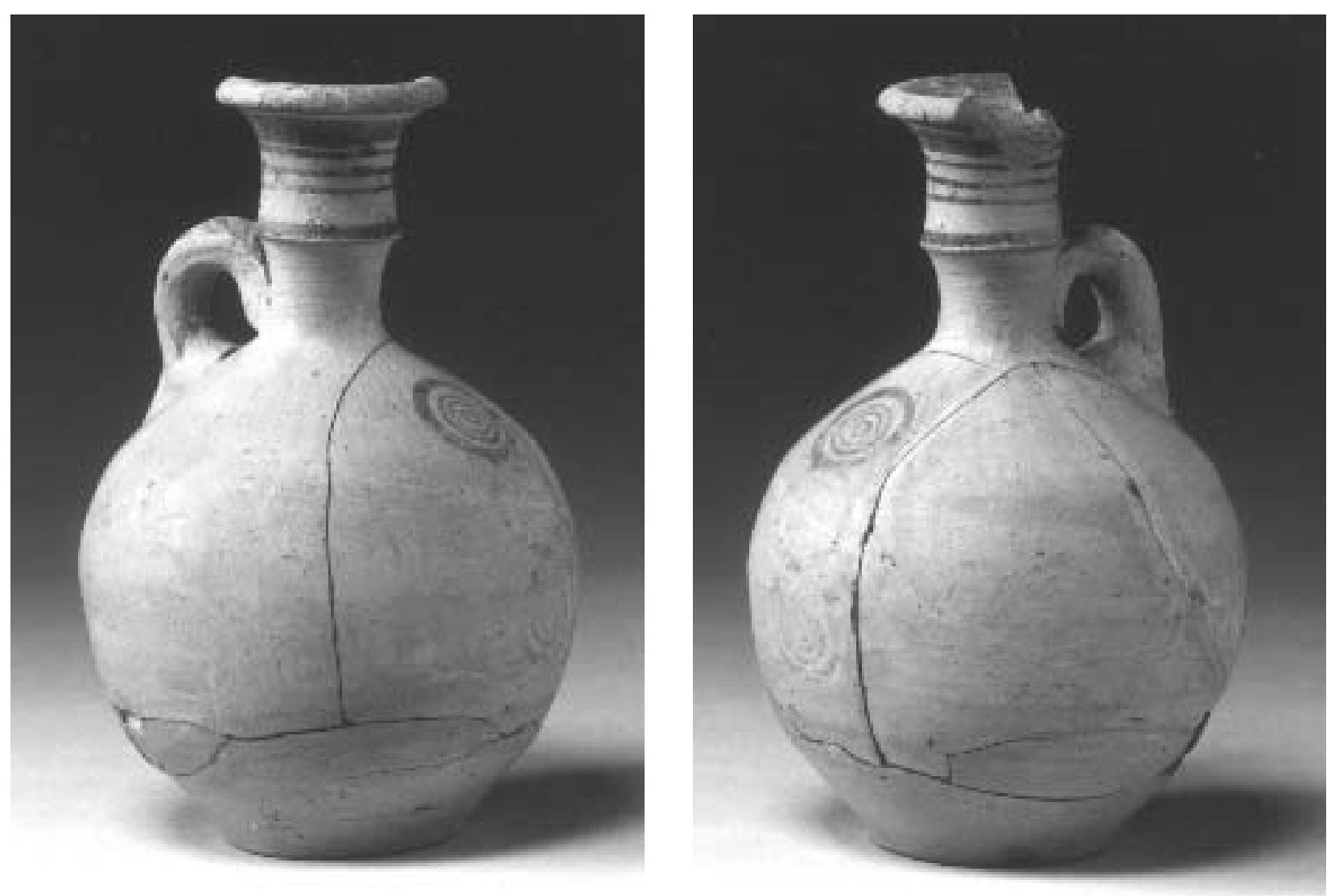

Abb. 6 Zypro-phönizisches Kännchen, Ägyptisches Museum Berlin, Inv. Nr. 7851. Foto: M. Büsing

36 Inv. Nr. 7851 und 7852 aus Grab 1198, Inv. Nr. 7871 aus Grab 866. Maße: Inv. Nr. 7851: $\mathrm{H}=10,9 \mathrm{~cm}, \varnothing \max .6,8$ cm. Inv. Nr. 7852: $\mathrm{H}=11,63 \mathrm{~cm}, \varnothing \max .6,3 \mathrm{~cm}$. Inv. Nr. 7871: $\mathrm{H}=8,9 \mathrm{~cm}, \varnothing \max .5,3 \mathrm{~cm}$. Parallelen dazu z. B. Land der Bibel 1997: 84-85, Kat. Nr. 133, 135-137; Moscati 1988: Abb. auf S. 235, 493, ähnlich auch 501; HARDEN 1962: pl. 112a. Siehe auch die in Ägypten gefundene Kännchen, z. B. aus Shaganbeh (AstoN 1996: 29, fig. 54.32) oder aus Theben (Aston 1996: 48, fig. 150.350).

37 Nach Harden (1962: Beschreibung zu pl. 112a) sind diese Gefäße in die Zeit des 7./6. Jh. v. Chr. zu datieren; dieser Typ wurde auf nahezu allen phönizischen und punischen Ausgrabungsstätten dieser Zeit gefunden. Zum mushroom-lipped jug siehe auch BIKAI 1987: 48-49.

38 7851: Lippe Typ 2, Hals Typ 1c, Körper Typ 1, Boden Typ 4, Dekoration Typ 2. 7852: Lippe Typ 1, Hals Typ 3a, Körper Typ 3a, Boden Typ 1. 7871: Lippe Typ 1, Hals Typ 5c, Körper Typ 1, Boden Typ 1. Siehe PESERICO 1996: 41, fig. 1 (Lippe), 42, fig. 2 (Hals), 42, fig. 3 (Körper), 42, fig. 4 (Boden). 

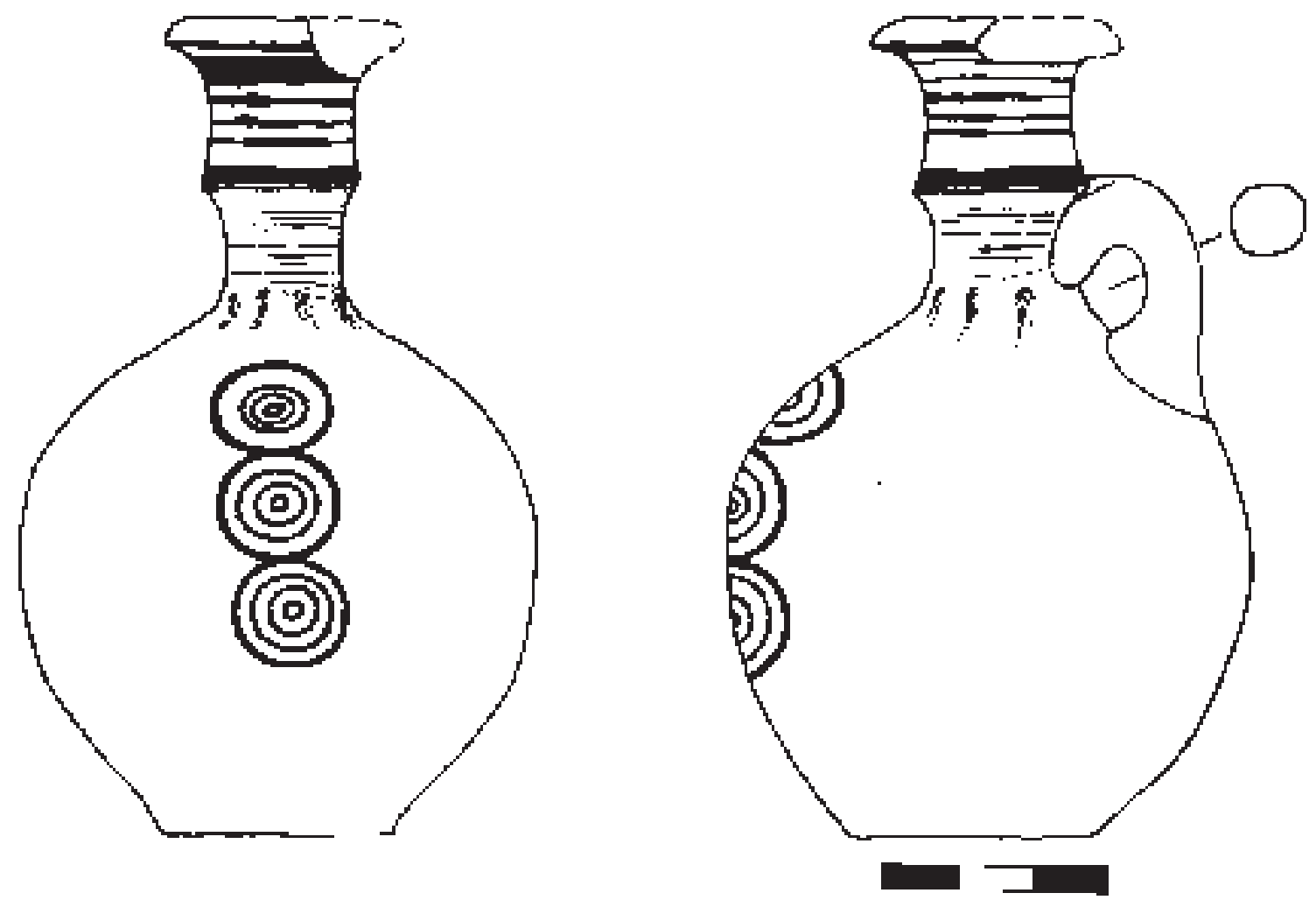

Abb. 7 Zypro-phönizisches Kännchen, Ägyptisches Museum Berlin, Inv. Nr. 7851. Zeichnung: A. Lohwasser, I. Säuberlich
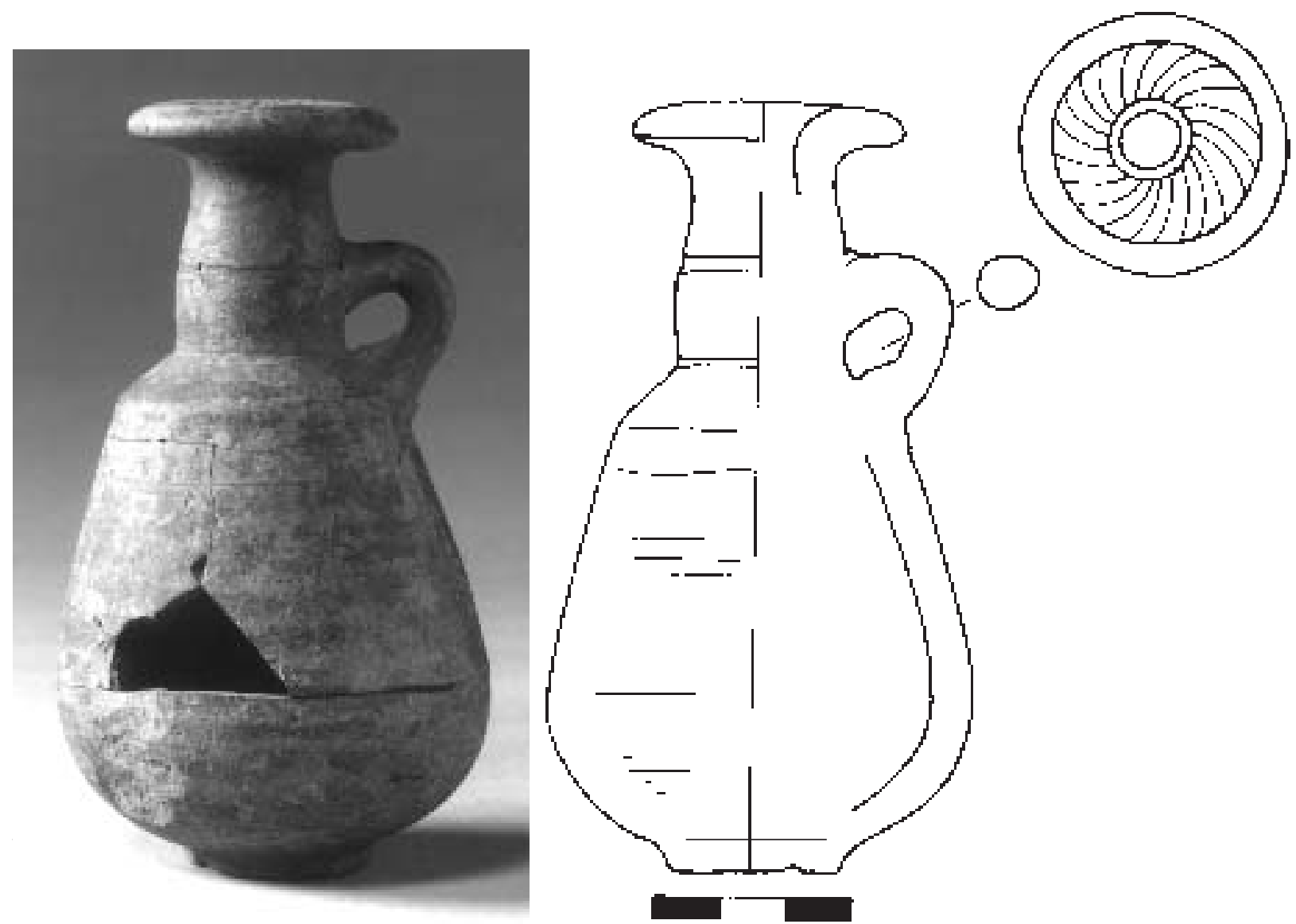

Abb. 8 Phönizisches Pilzkännchen, Ägyptisches Museum Berlin, Inv. Nr. 7852.

Foto: M. Büsing, Zeichnung: A. Lohwasser, I. Säuberlich 

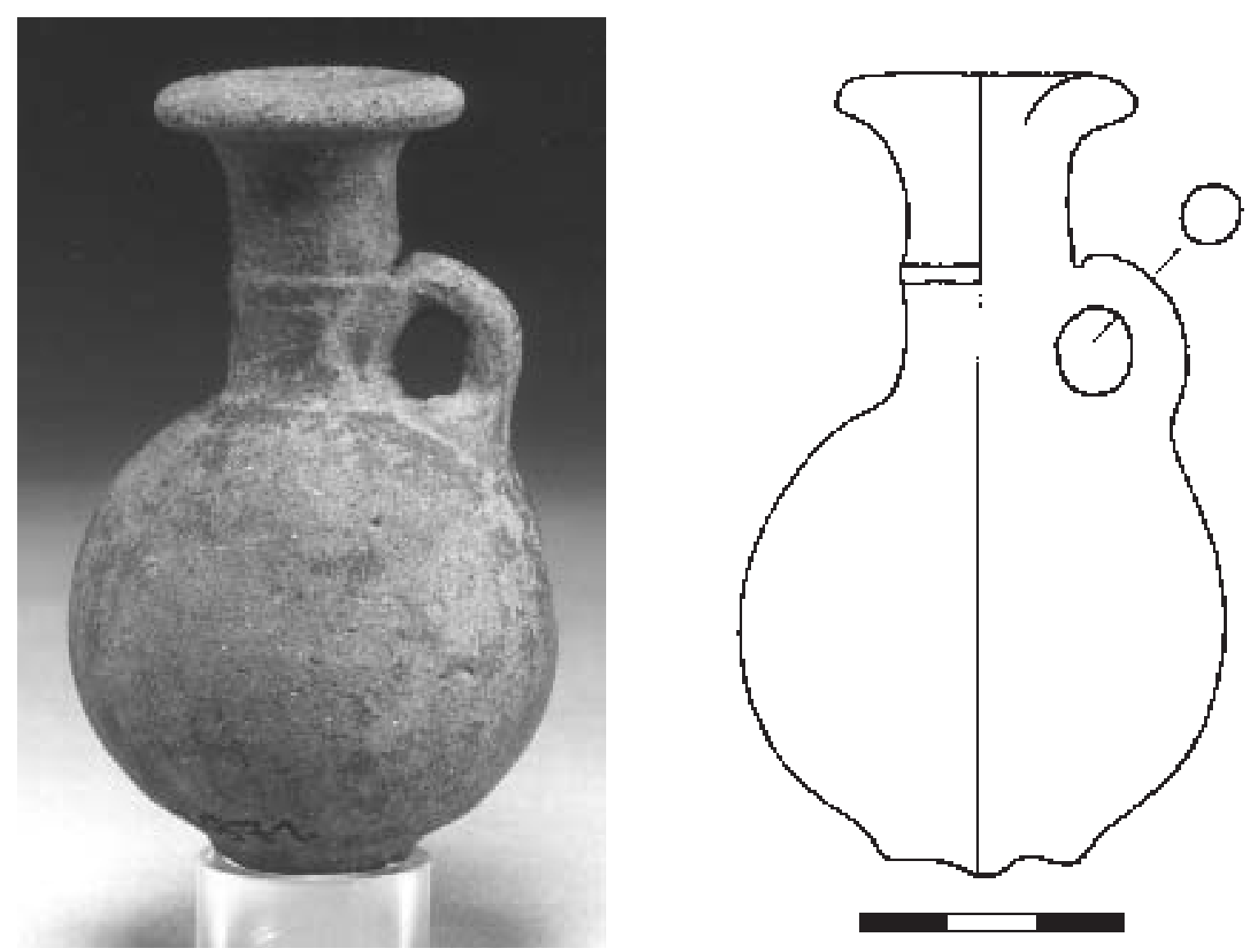

Abb. 9 Phönizisches Pilzkännchen, Ägyptisches Museum Berlin, Inv. Nr. 7871.

Foto: M. Büsing, Zeichnung: A. Lohwasser, I. Säuberlich

schwarze Bemalung von jeweils vier ineinanderliegenden Kreisen, die dreimal untereinander dargestellt ist. ${ }^{39}$ Der Hals des Gefäßes, der in der Mitte eine ringförmige Halsrippe hat, ist mit drei feinen schwarzen Linien am oberen Teil und einer breiten schwarzen Linie an der Halsrippe verziert. Die ausladende Lippe trägt auf der Außenseite eine breite rote Linie, eine ebensolche ist am Übergang von Gefäßhals zu Lippe. Die Innenseite der Lippe und des Halses sind, soweit sichtbar, mit roter Farbe bemalt. $^{40}$

Des weiteren wurden auch bei anderen Ausgrabungen in Kusch phönizische Objekte gefunden: ${ }^{41}$
In Hillat El-Arab, etwa gegenüber von Sanam auf der anderen Nilseite, konnten phönizische Gefäße geborgen werden, ${ }^{42}$ in El Kurru Scherben von ähnlichen phönizischen Amphoren. ${ }^{43}$ In Ku. 52, einem Grab, das in die Zeit des Königs Piye zu datieren ist, wurde ein wahrscheinlich ebenfalls phönizisches Krüglein gefunden. ${ }^{44}$ Sicherlich befinden sich auch unter den noch nicht bearbeiteten Keramikfragmenten aus El Kurru und Nuri weitere phönizische Scherben. Auch in Meroe wurde ein Gefäß aus Phönizien gefunden. ${ }^{45}$ Wieweit die vorwiegend im Grab des Aspelta ( $\mathrm{Nu}$. 8) gefundenen zylinderförmigen Behältnisse aus Goldblech mit den phönizischen

39 Das Motiv der ineinanderliegenden Kreise ist auf verschiedenen Gefäßtypen aus Zypern häufig, siehe BIKAI 1987: pl. II-IX; COLDSTREAM 1968: 276.

40 Parallele z. B. HARdEN 1962: pl. 111b. Er datiert das auf Zypern gefundene Kännchen in das 5. Jh. v. Chr.

41 Die Aufzählung erhebt keinen Anspruch auf Vollständigkeit!

\footnotetext{
42 Vincentelli 1996.

43 HEIDORN 1994: 124.

44 19-3-1054, Dunham 1950: 82, fig. 28c, pl. XLIII.B

45 ShinNIE \& BRADLEY 1980: 134 (P. 112), 107, fig. 37:101
} 
Amulettkapseln zu vergleichen sind, kann hier nicht untersucht werden. ${ }^{46}$

Die Beziehungen zwischen Kusch und der Mittelmeerwelt, insbesonders den Phöniziern, wurden bereits verschiedentlich untersucht. ${ }^{47}$ Der Handel mit Luxusgütern - Elfenbein, Gold, Exotica aus Afrika; Wein, Öl, Kunsthandwerk aus Vorderasien florierte besonders seit dem Neuen Reich, wobei eine Vielzahl von Gütern der Mittelmeerwelt nach Ägypten gelangten. Ab der 3. Zwischenzeit häufen sich die Funde aus dieser Region in Kusch, möglicherweise Belege dafür, daß der Handel nun direkt mit Kusch betrieben wurde. ${ }^{48}$ MоRкот (1995: 184-185) zählt eine Reihe von Indizien für den eigenständigen Handel zwischen Kusch und den Mittelmeerstaaten auf, wobei er seinen Schwerpunkt allerdings auf die von Kusch exportierten Güter - allen voran Elfenbein - legt. Die Darstellun-

Bibliographie

Aston, D.A.

1996 Egyptian Pottery of the Late New Kingdom and Third Intermediate Period (Twelfth - Seventh Centuries BC). Tentative Footsteps in a Forbidden Terrain, SAGA 13

Atasoy, E. and BuluÇ, S.,

1982 Metallurgical and archaeological examination of Phrygian Objects, Anatolian Studies 32, 157-160

BARNETT, R.D.,

1976 Sculptures from the North Palace of Assurbanipal at Niniveh (668-627 B.C.), London

BERENGUER, F.

1999 The Recent Discovery of a New Royal Pyramid in the Necropolis of Gebel Barkal (Kareima-Sudan), in: D. Welsby (ed.), Recent Research in Kushite History and Archaeology. Proceedings of the $8^{\text {th }}$ International Conference for Meroitic Studies (= British Museum. Occasional Paper Number 131), London 1999, 55-63

BIKAI, P.M.

1987 The Phoenician Pottery of Cyprus, Nicosia

BIRCH, W.D.G. and PINCHES, T.G.

1902 The Bronze Ornaments of the Palace Gates of Balawat (Shalmaneser II. B.C. 859-825), London

Bissing, Fr.W.v.

1923/24 Untersuchungen über die „phoinikischen“ Metallschalen, Jahrbuch DAI 37/9 (1923/24): 180-241

${ }^{46}$ Publikation der Grabfunde bei Dunham 1955: pl. XCIII-CXI. Zu der Funktion als Amulettkapseln äußert sich HölBL 1996: 346-347.

47 Morkot 1991: 329-332; MоRкот 1995: bes. 184-186; HEIDORN 1994: 127-131; LECLANT 1995: 45-46; VINCENTELLI 1996: 1043-1044. gen von Kuschiten mit innerafrikanischen Produkten in Persien zeigt, daß dieser Güteraustausch während der ganzen Spätzeit weiterbestand. ${ }^{49}$ Handelsware, die aus dem Vorderen Orient nach Kusch importiert wurde, ist bis jetzt erst in geringem Umfang bekannt. Es ist allerdings anzunehmen, daß die hier vorgestellten Objekte nur ein kleiner Teil des tatsächlich vorhandenen importierten Materials darstellen. Es ist dabei auch an eine Route über das Rote Meer, unter Ausschluß Ägyptens als Zwischenhändler, zu denken. Die Bronzeschale sowie die drei Keramikgefäße, die ursprünglich aus dem phönizischen Kulturraum stammen und eine tausende Kilometer lange Reise bis zum Friedhof von Sanam im Sudan zurückgelegt haben, können wohl in den Kontext „Austausch von Luxusgütern“ gestellt werden und sind ein Teil eines ständigen Handelsflusses zwischen Kusch und der Mittelmeerwelt.

Brewer, D.J., REDFORD, D.B. and REDFORD, S.

1994 Domestic Plants and Animals. The Egyptian Origins, Warminster

BudGE, E.A.W.

1914 Assyrian Sculptures in the British Museum. Reign of Assur-nasir-pal, 885-860 B.C., London

Coldstream, J.N.

1968 Greek Geometric Pottery. A Survey of Ten Local Styles and their Chronology, London

Culcian, W.

1970 Coupes a décor phénicien provenant d'Iran, Syria 47, 65-76

DUNHAM, D.

1950 El Kurru. The Royal Cemeteries of Kush I., Boston

1955 Nuri. The Royal Cemeteries of Kush II., Boston

EDWARDS, D.N.

1996 The Archaeology of the Meroitic State. New Perspectives on its Social and Political Organisation, BAR Internat. Series 640, Oxford

FALSONE, G.

1985 A Syro-Phoenician Bull-Bowl in Geneva and its Analogue in the British Museum, Anatolian Studies 35, 131-142

1988 Phoenicia as a Bronzeworking Centre in the Iron Age, in: J. CurTis (ed.), Bronze-working Centers of

\footnotetext{
48 Da die Phönizier als bedeutendste Seefahrer der Alten Welt gelten, kann angenommen werden, daß Handelskontakte über den Seeweg (Rotes Meer) bestanden. Die Region ist allerdings bis jetzt archäologisch kaum untersucht.

49 Morkot 1991: 324-325.
} 
Western Asia, c.1000-539 B.C., London / New York, 227-250

FriedmanN, F.D. (Hg.)

1998 Gifts of the Nile. Ancient Egyptian Faience, Rhode Island

GRIFFITH, F.LL.

1922 Oxford Excavations in Nubia, LAAA 9, 67-124

1923 Oxford Excavations in Nubia, LAAA 10, 73-171

GRIMAL, N.-C.

1981 La stèle triomphale de Pi('ankhy) au Musée du Caire JE 48862 et 47086-47089, Kairo

HARDEN, D.

1962 The Phoenicians, London

HEIDORN, L.

1994 Historical Implications of the Pottery from the Earliest Tombs at El-Kurru, JARCE 31 : 115-131

HermanN, G.

1992 The Small Collections from Fort Shalmaneser. Ivories from Nimrud V, London

Hintze, F. (Hg.)

1971 Musawwrat es Sufra I,2. Der Löwentempel (Tafelband), Berlin

Hofmann, I. and Tomand, $\mathrm{H}$.

1987 Die Bedeutung des Tieres in der meroitischen Kultur, BzS Beiheft 2

Hogarth, D.G.

1922 Engraved Hittite Objects, JEA 8, 211-218

HölBL, G.

1979 Beziehungen der ägyptischen Kultur zu Altitalien. Études préliminaires aux religions orientales dans l'empire romain 62

1996 Ägyptisches Kulturgut im phönikischen und punischen Sardinien. Études préliminaires aux religions orientales dans l'empire romain 102

Kendall, T.

1986 Gebel Barkal Epigraphic Survey: 1986. Preliminary Report of First Season's Activity. Observing the Cliff Inscription and Bridge Emplacement, and Recovering the Piye Reliefs in the Great Amun Temple, Boston

SEIPEL, W. (Hg.)

1997 Land der Bibel. Schätze aus dem Israel Museum Jerusalem, Wien

LAYARD, A.H.

1853 A Second Series of the Monuments of Niniveh, London 1853

LECLANT, J.

1995 Carthage et l'Égypte, in: M.H. FAnTAR and M. GHAKI (Hg.), Actes du III congrès international des études phéniciennes et puniques, Vol. I, Tunis, 41-50

Löw, U.

1998 Figürlich verzierte Metallgefäße aus Nord- und Nordwestiran, AVO 6

MARKoe, G.

1985 Phoenician Bronze and Silver Bowls from Cyprus and the Mediterranean, Berkley

MOOREY, P.R.S.

1971 Catalogue of the Ancient Persian Bronzes in the Ashmolean Museum, Oxford
Morkot, R.

1991 Nubia and Achaemenid Persia: Sources and Problems, in: H. SAncisi-Weerdenburg and A. KuhrT (Hg.), Achaemenid History VI: Asia Minor and Egypt: Old Cultures in a New Empire. Proceedings of the Groningen 1988 Achaemenid History Workshop, Leiden, 321-335

1995 The economy in Nubia in the New Kingdom, in: Actes de la VIII Conférence Internationale des Études Nubiennes, Lille 11-17 septembre 1994, Vol. I - Communications principales (=CRIPEL 17/1), 175-189

Moscati, S. (ed.),

1988 The Phoenicians. Katalog zur Ausstellung im Palazzo Grassi, Venedig

Muscarella, O.W. (ed.)

1974 Ancient Art. The Norbert Schimmel Collection, Mainz

Negahban, E.O.

1983 Metal Vases from Marlik, PBF II/3

ONASCH, CH.

1993 Die religiöse Bedeutung des Tempels, in: F. Hintze et al., Musawwarat es Sufra I,1. Der Löwentempel (Textband), Berlin, 228-267

ONASCH, H.-U.

1994 Die assyrischen Eroberungen Ägyptens, ÄUAT 27

Osborne, J. and OsBornová, J.

1998 The Mammals of Ancient Egypt, Warminster

PALEy, S.M. and Sobolewski, R.P.

1987 The reconstruction of the Relief Representations and their Positions in the Northwest-Palace at Kalhu (Nimrud) II, Baghdader Forschungen 10

PESERICO, A.

1996 Le brocche „a fungo“ fenice nel mediterraneo. Tipologia e cronologia, Rom

READE, J.E.

1985 Texts and Sculpture from the North-West Palace, Nimrud, Iraq 47, 203-214

SHINNIE, P.L. and BRADLEY, R.J.

1980 The Capital of Cush I. Meroe Excavations 1965-1972, Meroitica 4

VINCENTELLI, I.

1996 Due anfore „fenicie“ nella necropoli Kushita di Hillat el Arab (Sudan), in: E. AcQuaro, Alle Goglie della classicità il mediterranoe tra traduzione e innovazione. Studi in onore di Sabatino Moscati, Vol II, PisaRom, 1036-1044

VinCENTELLI-LiVERANI, I.

1997 Recent Excavations in the Napatan Cemetery of Hillat el Arab, CRIPEL 17/3, 119-127

WALLIS, H.

1898 Egyptian ceramic art. The MacGregor Collection, o.O.

WeLSBY, D.

1996 The Kingdom of Kush. The Napatan and Meroitic Empires, London

WENIG, ST.

1993 Die Darstellungen. Untersuchungen zu Ikonographie, Inhalt und Komposition der Reliefs, in: F. Hintze et al., Musawwarat es Sufra I,1. Der Löwentempel (Textband), Berlin, 74-227 